\title{
Global smooth solutions to 3D MHD with mixed partial dissipation and magnetic diffusion
}

Menglong $\mathrm{Su}^{1,2^{*}}$ and Jian Wang ${ }^{3}$

\section{"Correspondence:}

mlsulynu@163.com

'School of Mathematical Sciences,

Luoyang Normal University,

Luoyang, 471022, P.R. China

${ }^{2}$ Key Laboratory of Symbolic

Computation and Knowledge

Engineering of Ministry of

Education, Jilin University,

Changchun, 130012, P.R. China

Full list of author information is

available at the end of the article

\begin{abstract}
In this paper, we prove the existence of global smooth solutions to the Cauchy problem of 3D incompressible magnetohydrodynamics (MHD) flows with mixed partial dissipation and magnetic diffusion if the initial condition is suitably small.

Keywords: incompressible magnetohydrodynamics; global smooth solution; partial dissipation and magnetic diffusion
\end{abstract}

\section{Introduction}

In this paper, we consider the following 3D incompressible MHD equations with mixed partial dissipation and magnetic diffusion (see [1]), i.e.,

$$
\begin{aligned}
& u_{t}+u \cdot \nabla u=-\nabla p+\mu u_{x x}+\mu u_{y y}+b \cdot \nabla b, \\
& b_{t}+u \cdot \nabla b=\eta b_{x x}+\eta b_{y y}+b \cdot \nabla u \\
& \operatorname{div} u=0, \quad \operatorname{div} b=0
\end{aligned}
$$

associated with the initial data

$$
u(0, x)=u_{0}, \quad b(0, x)=b_{0} .
$$

Here $u=\left(u_{1}(t, x), u_{2}(t, x), u_{3}(t, x)\right)$ is the velocity field, $b=\left(b_{1}(t, x), b_{2}(t, x), b_{3}(t, x)\right)$ is the magnetic field, $p=p(t, x)$ is scalar pressure, $\mu>0$ is the kinematic viscosity, $\eta>0$ is the magnetic diffusion. For more background, we refer the reader to [2] for MHD and [1,3] for MHD with mixed partial dissipation and magnetic diffusion. Without loss of generality, we assume that $\mu=\eta=1$ in the remainder of the paper.

To state the main results, we first introduce the following conventions and notations which will be used throughout this paper. Set

$$
\int f \mathrm{~d} x \triangleq \int_{\mathbb{R}^{3}} f \mathrm{~d} x \mathrm{~d} y \mathrm{~d} z,
$$

○ 2013 Su and Wang; licensee Springer. This is an Open Access article distributed under the terms of the Creative Commons Attribution License (http://creativecommons.org/licenses/by/2.0), which permits unrestricted use, distribution, and reproduction in any medium, provided the original work is properly cited. 
and that $\|\cdot\|$ is the $L^{2}$ norm, i.e.,

$$
\begin{aligned}
& \|f\|=\left(\int f^{2} \mathrm{~d} x\right)^{\frac{1}{2}}, \\
& H^{m} \triangleq W^{m, 2}\left(\mathbb{R}^{3}\right)=\left\{\sum_{|\alpha| \leq m} \int\left|D^{\alpha} u\right|^{2} \mathrm{~d} x\right\}^{1 / 2} \text { with the norm }\|\cdot\|_{H^{m}} .
\end{aligned}
$$

Our main result of this paper can be stated as follows.

Theorem 1.1 Assume that $u_{0} \in H^{2}$ and $b_{0} \in H^{2}$ with $\operatorname{div} u_{0}=\operatorname{div} b_{0}=0$ and $\left\|u_{0}\right\|_{H^{1}}+$ $\left\|b_{0}\right\|_{H^{1}} \leq \varepsilon$, where $\varepsilon$ is a sufficiently small positive number. Then (1)-(4) admit global smooth solutions.

Remark 1.1 Theorem 1.1 is Theorem 1.2 in [1], which has not been proved in their paper. We would also emphasize that our proof of Theorem 1.1 is clearer for deducing the desired a priori estimates in Lemma 2.3 (see the next section) than that of Proposition 3.1 in [1].

The rest of the paper is organized as follows. In Section 2, we deduce the desired $a$ priori estimates to complete the proof of Theorem 1.1. We finish the proof of Theorem 1.1 in Section 3 by the method of vanishing viscosities.

\section{A priori estimates}

In this section, we deduce the desired a priori estimates in order to finish the main result. Before we begin to prove the main theorem of this paper, we first state the following useful lemma that was deduced in [1].

Lemma 2.1 Assume that $f, g, h, f_{x}, g_{y}, h_{z}$ are all in $L^{2}\left(\mathbb{R}^{3}\right)$. Then we have

$$
\int|f g h| \mathrm{d} x \leq C\|f\|^{\frac{1}{2}}\|g\|^{\frac{1}{2}}\|h\|^{\frac{1}{2}}\left\|f_{x}\right\|^{\frac{1}{2}}\left\|g_{y}\right\|^{\frac{1}{2}}\left\|h_{z}\right\|^{\frac{1}{2}}
$$

Clearly, the standard energy estimate shows that

$$
\frac{1}{2} \frac{\mathrm{d}}{\mathrm{d} t}\left(\|u\|^{2}+\|b\|^{2}\right)+\left\|u_{x}\right\|^{2}+\left\|u_{y}\right\|^{2}+\left\|b_{x}\right\|^{2}+\left\|b_{y}\right\|^{2}=0 .
$$

We denote that $\omega=\nabla \times u$ and $j=\nabla \times b$. Thus, applying the operator ' $\nabla \times$ ' to (1) and (2), together with (3), we deduce that

$$
\begin{aligned}
& \omega_{t}+u \cdot \nabla \omega-\omega \cdot \nabla u=\omega_{x x}+\omega_{y y}+b \cdot \nabla j-j \cdot \nabla b, \\
& j_{t}+u \cdot \nabla j=j_{x x}+j_{y y}+b \cdot \nabla \omega+\varepsilon_{i j k}\left(\partial_{j} b_{l} \partial_{l} u_{k}-\partial_{j} u_{l} \partial_{l} b_{k}\right),
\end{aligned}
$$

where $\varepsilon_{i j k}$ is defined as follows:

$$
\varepsilon_{i j k}= \begin{cases}1 & \text { if }(i, j, k) \text { is an even permutation, } \\ -1 & \text { if }(i, j, k) \text { is an odd permutation, } \\ 0 & \text { others. }\end{cases}
$$


The first key lemma is the following.

Lemma 2.2 If $(u, b)$ solves (1)-(4) and the initial data satisfies

$$
\left\|u_{0}\right\|^{2}+\left\|b_{0}\right\|^{2} \leq \frac{1}{4 C} \quad \text { and } \quad\left\|\omega_{0}\right\|^{2}+\left\|j_{0}\right\|^{2} \leq \frac{1}{4}
$$

where $C$ is a suitable large number, then the vorticity $\omega$ and the current density $j$ satisfy

$$
\|\omega\|^{2}+\|j\|^{2} \leq 1, \quad \int_{0}^{t}\left(\left\|\omega_{x}\right\|^{2}+\left\|\omega_{y}\right\|^{2}+\left\|j_{x}\right\|^{2}+\left\|j_{y}\right\|^{2}\right) \mathrm{d} s \leq 1 \quad \text { for all } t \geq 0 .
$$

Proof Multiplying (6) and (7) by $\omega$ and $j$, respectively, then integrating the resulting equations by parts, after adding the two equalities together, we finally deduce

$$
\begin{aligned}
& \frac{1}{2} \frac{\mathrm{d}}{\mathrm{d} t}\left(\|\omega\|^{2}+\|j\|^{2}\right)+\left\|\omega_{x}\right\|^{2}+\left\|\omega_{y}\right\|^{2}+\left\|j_{x}\right\|^{2}+\left\|j_{y}\right\|^{2} \\
& \quad=\int\left(\omega \cdot \nabla \omega \cdot \omega-j \cdot \nabla b \cdot \omega+\varepsilon_{i j k}\left(\partial_{j} b_{l} \partial_{l} u_{k}-\partial_{j} u_{l} \partial_{l} b_{k}\right) j_{i}\right) \mathrm{d} x=I+J+K+L .
\end{aligned}
$$

We have to estimate each term on the right-hand side of (9). Some of the terms are the same as in [1] and are proved here for completeness. First, $I$ can be written as

$$
\begin{aligned}
I & =\int \omega \cdot \nabla u \cdot \omega \mathrm{d} x=\int \omega_{i} \partial_{i} u_{j} \omega_{j} \mathrm{~d} x \\
& =\int \omega_{1} \partial_{x} u_{j} \omega_{j} \mathrm{~d} x+\int \omega_{2} \partial_{y} u_{j} \omega_{j} \mathrm{~d} x+\int \omega_{3} \partial_{z} u_{j} \omega_{j} \mathrm{~d} x=I_{1}+I_{2}+I_{3} .
\end{aligned}
$$

With the help of Lemma 2.1, we deduce that

$$
\begin{aligned}
I_{1} & =\int \omega_{1} \partial_{x} u_{j} \omega_{j} \mathrm{~d} x \leq C\|\omega\|^{\frac{1}{2}}\left\|u_{x}\right\|^{\frac{1}{2}}\|\omega\|^{\frac{1}{2}}\left\|\omega_{x}\right\|^{\frac{1}{2}}\left\|\omega_{y}\right\|^{\frac{1}{2}}\left\|\omega_{x}\right\|^{\frac{1}{2}} \\
& \leq \frac{1}{24}\left\|\omega_{x}\right\|^{2}+\frac{1}{16}\left\|\omega_{y}\right\|^{2}+C\left\|u_{x}\right\|^{2}\|\omega\|^{4} .
\end{aligned}
$$

Similarly, we obtain that

$$
I_{2}=\int \omega_{2} \partial_{y} u_{j} \omega_{j} \mathrm{~d} x \leq \frac{1}{24}\left\|\omega_{x}\right\|^{2}+\frac{1}{16}\left\|\omega_{y}\right\|^{2}+C\left\|u_{y}\right\|^{2}\|\omega\|^{4},
$$

and

$$
\begin{aligned}
I_{3} & =\int \omega_{3} \partial_{z} u_{j} \omega_{j} \mathrm{~d} x=\int\left(\partial_{x} u_{2}-\partial_{y} u_{1}\right) \partial_{z} u_{j} \omega_{j} \mathrm{~d} x \\
& \leq \frac{1}{24}\left\|\omega_{x}\right\|^{2}+\frac{1}{16}\left\|\omega_{y}\right\|^{2}+C\left(\left\|u_{x}\right\|^{2}+\left\|u_{y}\right\|^{2}\right)\|\omega\|^{4} .
\end{aligned}
$$

In order to bound $J$, we rewrite the integrand explicitly as follows:

$$
\begin{aligned}
J & =-\int j \cdot \nabla b \cdot \omega \mathrm{d} x=-\int j_{i} \partial_{i} b_{j} \omega_{j} \mathrm{~d} x \\
& =-\int j_{1} \partial_{x} b_{j} \omega_{j} \mathrm{~d} x-\int j_{2} \partial_{y} b_{j} \omega_{j} \mathrm{~d} x-\int j_{3} \partial_{z} b_{j} \omega_{j} \mathrm{~d} x=J_{1}+J_{2}+J_{3} .
\end{aligned}
$$


Due to Lemma 2.1, we see that

$$
\begin{aligned}
J_{1} & =-\int j_{1} \partial_{x} b_{j} \omega_{j} \mathrm{~d} x \leq C\|j\|^{\frac{1}{2}}\left\|b_{x}\right\|^{\frac{1}{2}}\|\omega\|^{\frac{1}{2}}\left\|j_{x}\right\|^{\frac{1}{2}}\left\|\partial_{x} b_{z}\right\|^{\frac{1}{2}}\left\|\omega_{y}\right\|^{\frac{1}{2}} \\
& \leq \frac{1}{22}\left\|j_{x}\right\|^{2}+\frac{1}{16}\left\|\omega_{y}\right\|^{2}+C\left\|b_{x}\right\|^{2}\left(\|\omega\|^{4}+\|j\|^{4}\right) .
\end{aligned}
$$

Similarly,

$$
\begin{aligned}
J_{2} & =-\int j_{2} \partial_{y} b_{j} \omega_{j} \mathrm{~d} x \leq \frac{1}{16}\left\|\omega_{y}\right\|^{2}+\frac{1}{22}\left\|j_{x}\right\|^{2}+\frac{1}{26}\left\|j_{y}\right\|^{2}+C\left\|b_{y}\right\|^{2}\left(\|\omega\|^{4}+\|j\|^{4}\right), \\
J_{3} & =-\int j_{3} \partial_{z} b_{j} \omega_{j} \mathrm{~d} x=-\int\left(\partial_{x} b_{2}-\partial_{y} b_{1}\right) \partial_{z} b_{j} \omega_{j} \mathrm{~d} x \\
& \leq \frac{1}{24}\left\|\omega_{x}\right\|^{2}+\frac{1}{22}\left\|j_{x}\right\|^{2}+\frac{1}{26}\left\|j_{y}\right\|^{2}+C\left(\left\|b_{x}\right\|^{2}+\left\|b_{y}\right\|^{2}\right)\left(\|\omega\|^{4}+\|j\|^{4}\right) .
\end{aligned}
$$

Now, let us turn to bound $L$,

$$
\begin{aligned}
L & =-\int \varepsilon_{i j k} \partial_{j} u_{l} \partial_{l} u_{k} j_{i} \mathrm{~d} x \\
& =-\int \varepsilon_{i j k} \partial_{x} u_{l} \partial_{l} u_{k} j_{i} \mathrm{~d} x-\int \varepsilon_{i j k} \partial_{y} u_{l} \partial_{l} u_{k} j_{i} \mathrm{~d} x-\int \varepsilon_{i j k} \partial_{z} u_{l} \partial_{l} u_{k} j_{i} \mathrm{~d} x \\
& =L_{1}+L_{2}+L_{3} .
\end{aligned}
$$

By Lemma 2.1, we have that

$$
\begin{aligned}
L_{1} & =-\int \varepsilon_{i j k} \partial_{x} u_{l} \partial_{l} u_{k} j_{i} \mathrm{~d} x \leq C\left\|u_{x}\right\|^{\frac{1}{2}}\|j\|^{\frac{1}{2}}\|j\|^{\frac{1}{2}}\left\|j_{x}\right\|^{\frac{1}{2}}\left\|j_{y}\right\|^{\frac{1}{2}}\left\|\omega_{x}\right\|^{\frac{1}{2}} \\
& \leq \frac{1}{24}\left\|\omega_{x}\right\|^{2}+\frac{1}{22}\left\|j_{x}\right\|^{2}+\frac{1}{26}\left\|j_{y}\right\|^{2}+C\left\|u_{x}\right\|^{2}\|j\|^{4} .
\end{aligned}
$$

Similarly,

$$
L_{2}=-\int \varepsilon_{i j k} \partial_{y} u_{l} \partial_{l} u_{k} j_{i} \mathrm{~d} x \leq \frac{1}{16}\left\|\omega_{y}\right\|^{2}+\frac{1}{22}\left\|j_{x}\right\|^{2}+\frac{1}{26}\left\|j_{y}\right\|^{2}+C\left\|u_{y}\right\|^{2}\|j\|^{4} .
$$

As for $L_{3}$, we should split it into three parts:

$$
\begin{aligned}
L_{3} & =-\int \varepsilon_{i j k} \partial_{z} u_{l} \partial_{l} u_{k} j_{i} \mathrm{~d} x \\
& =-\int \varepsilon_{i j k} \partial_{z} u_{1} \partial_{x} u_{k} j_{i} \mathrm{~d} x-\int \varepsilon_{i j k} \partial_{z} u_{2} \partial_{y} u_{k} j_{i} \mathrm{~d} x-\int \varepsilon_{i j k} \partial_{z} u_{3} \partial_{z} u_{k} j_{i} \mathrm{~d} x \\
& =L_{31}+L_{32}+L_{33}, \\
L_{31} & =-\int \varepsilon_{i j k} \partial_{z} u_{1} \partial_{x} u_{k} j_{i} \mathrm{~d} x \\
& \leq C\|\omega\|^{\frac{1}{2}}\left\|b_{x}\right\|^{\frac{1}{2}}\|j\|^{\frac{1}{2}}\left\|\omega_{x}\right\|^{\frac{1}{2}}\left\|\partial_{x} b_{z}\right\|^{\frac{1}{2}}\left\|j_{y}\right\|^{\frac{1}{2}} \\
& \leq \frac{1}{24}\left\|\omega_{x}\right\|^{2}+\frac{1}{22}\left\|j_{x}\right\|^{2}+\frac{1}{26}\left\|j_{y}\right\|^{2}+C\left\|b_{x}\right\|^{2}\left(\|\omega\|^{4}+\|j\|^{4}\right) .
\end{aligned}
$$


Similarly,

$$
L_{32}=-\int \varepsilon_{i j k} \partial_{z} u_{2} \partial_{y} u_{k} j_{i} \mathrm{~d} x \leq \frac{1}{24}\left\|\omega_{x}\right\|^{2}+\frac{1}{26}\left\|j_{y}\right\|^{2}+C\left\|b_{y}\right\|^{2}\left(\|\omega\|^{4}+\|j\|^{4}\right) .
$$

To bound $L_{33}$, using the incompressibility condition $\operatorname{div} u=0$, we deduce that

$$
\begin{aligned}
L_{33} & =-\int \varepsilon_{i j k} \partial_{z} u_{3} \partial_{z} u_{k} j_{i} \mathrm{~d} x \\
& =\int \varepsilon_{i j k}\left(\partial_{x} u_{1}+\partial_{y} u_{2}\right) \partial_{z} u_{k} j_{i} \mathrm{~d} x=L_{33}^{1}+L_{33}^{2} .
\end{aligned}
$$

We get the $L_{33}^{1}$ and $L_{33}^{2}$ as follows:

$$
\begin{aligned}
L_{33}^{1} & =\int \varepsilon_{i j k} \partial_{x} u_{1} \partial_{z} u_{k} j_{i} \mathrm{~d} x \leq C\left\|u_{x}\right\|^{\frac{1}{2}}\|j\|^{\frac{1}{2}}\|j\|^{\frac{1}{2}}\left\|\partial_{x} u_{z}\right\|^{\frac{1}{2}}\left\|j_{x}\right\|^{\frac{1}{2}}\left\|j_{y}\right\|^{\frac{1}{2}} \\
& \leq \frac{1}{24}\left\|\omega_{x}\right\|^{2}+\frac{1}{22}\left\|j_{x}\right\|^{2}+\frac{1}{26}\left\|j_{y}\right\|^{2}+C\left\|u_{x}\right\|^{2}\|j\|^{4},
\end{aligned}
$$

and

$$
L_{33}^{2} \leq \frac{1}{16}\left\|\omega_{y}\right\|^{2}+\frac{1}{22}\left\|j_{x}\right\|^{2}+\frac{1}{26}\left\|j_{y}\right\|^{2}+C\left\|u_{y}\right\|^{2}\|j\|^{4} .
$$

To bound $K$, we should divide it into three parts:

$$
\begin{aligned}
K & =\int \varepsilon_{i j k} \partial_{j} b_{l} \partial_{l} u_{k} j_{i} \mathrm{~d} x \\
& =\int \varepsilon_{i 1 k} \partial_{x} b_{l} \partial_{l} u_{k} j_{i} \mathrm{~d} x+\int \varepsilon_{i 2 k} \partial_{y} b_{l} \partial_{l} u_{k} j_{i} \mathrm{~d} x+\int \varepsilon_{i 3 k} \partial_{z} b_{l} \partial_{l} u_{k} j_{i} \mathrm{~d} x \\
& =K_{1}+K_{2}+K_{3} .
\end{aligned}
$$

Similarly, we deduce that

$$
\begin{aligned}
K_{1} & =\int \varepsilon_{i 1 k} \partial_{x} b_{l} \partial_{l} u_{k} j_{i} \mathrm{~d} x \leq C\left\|b_{x}\right\|^{\frac{1}{2}}\|\omega\|^{\frac{1}{2}}\|j\|^{\frac{1}{2}}\left\|\partial_{x} b_{z}\right\|^{\frac{1}{2}}\left\|\omega_{x}\right\|^{\frac{1}{2}}\left\|j_{y}\right\|^{\frac{1}{2}} \\
& \leq \frac{1}{24}\left\|\omega_{x}\right\|^{2}+\frac{1}{22}\left\|j_{x}\right\|^{2}+\frac{1}{26}\left\|j_{y}\right\|^{2}+C\left\|b_{x}\right\|^{2}\left(\|\omega\|^{4}+\|j\|^{4}\right),
\end{aligned}
$$

and

$$
K_{2}=\int \varepsilon_{i 2 k} \partial_{y} b_{l} \partial_{l} u_{k} j_{i} \mathrm{~d} x \leq \frac{1}{24}\left\|\omega_{x}\right\|^{2}+\frac{1}{26}\left\|j_{y}\right\|^{2}+C\left\|b_{y}\right\|^{2}\left(\|\omega\|^{4}+\|j\|^{4}\right) .
$$

For $K_{3}$, we have

$$
\begin{aligned}
K_{3} & =\int \varepsilon_{i 3 k} \partial_{z} b_{l} \partial_{l} u_{k} j_{i} \mathrm{~d} x \\
& =\int \varepsilon_{i 3 k} \partial_{z} b_{1} \partial_{x} u_{k} j_{i} \mathrm{~d} x+\int \varepsilon_{i 3 k} \partial_{z} b_{2} \partial_{y} u_{k} j_{i} \mathrm{~d} x+\int \varepsilon_{i 3 k} \partial_{z} b_{3} \partial_{z} u_{k} j_{i} \mathrm{~d} x \\
& =K_{31}+K_{32}+K_{33} .
\end{aligned}
$$


Thus,

$$
\begin{aligned}
K_{31} & =\int \varepsilon_{i 3 k} \partial_{z} b_{1} \partial_{x} u_{k} j_{i} \mathrm{~d} x \leq C\|j\|^{\frac{1}{2}}\left\|u_{x}\right\|^{\frac{1}{2}}\|j\|^{\frac{1}{2}}\left\|\omega_{x}\right\|^{\frac{1}{2}}\left\|j_{x}\right\|^{\frac{1}{2}}\left\|j_{y}\right\|^{\frac{1}{2}} \\
& \leq \frac{1}{24}\left\|\omega_{x}\right\|^{2}+\frac{1}{22}\left\|j_{x}\right\|^{2}+\frac{1}{26}\left\|j_{y}\right\|^{2}+C\left\|u_{x}\right\|^{2}\|j\|^{4}
\end{aligned}
$$

and

$$
K_{32}=\int \varepsilon_{i 3 k} \partial_{z} b_{2} \partial_{y} u_{k} j_{i} \mathrm{~d} x \leq \frac{1}{16}\left\|\omega_{y}\right\|^{2}+\frac{1}{22}\left\|j_{x}\right\|^{2}+\frac{1}{26}\left\|j_{y}\right\|^{2}+C\left\|u_{y}\right\|^{2}\|j\|^{4} .
$$

As for $K_{33}$, using $\operatorname{div} b=0$, we obtain

$$
\begin{aligned}
K_{33} & =\int \varepsilon_{i 3 k} \partial_{z} b_{3} \partial_{z} u_{k} j_{i} \mathrm{~d} x=-\int \varepsilon_{i 3 k}\left(\partial_{x} b_{1}+\partial_{y} b_{2}\right) \partial_{z} u_{k} j_{i} \mathrm{~d} x \\
& \leq \frac{1}{24}\left\|\omega_{x}\right\|^{2}+\frac{1}{26}\left\|j_{y}\right\|^{2}+C\left(\left\|b_{x}\right\|^{2}+\left\|b_{y}\right\|^{2}\right)\left(\|\omega\|^{4}+\|j\|^{4}\right) .
\end{aligned}
$$

Substituting all the above estimates into (9), we conclude that

$$
\begin{gathered}
\frac{1}{2} \frac{\mathrm{d}}{\mathrm{d} t}\left(\|\omega\|^{2}+\|j\|^{2}\right)+\frac{1}{2}\left(\left\|\omega_{x}\right\|^{2}+\left\|\omega_{y}\right\|^{2}+\left\|j_{x}\right\|^{2}+\left\|j_{y}\right\|^{2}\right) \\
\leq C\left(\left\|u_{x}\right\|^{2}+\left\|u_{y}\right\|^{2}+\left\|b_{x}\right\|^{2}+\left\|b_{y}\right\|^{2}\right)\left(\|\omega\|^{4}+\|j\|^{4}\right) .
\end{gathered}
$$

Let $\|\omega\|^{2}+\|j\|^{2} \leq 1$, we deduce, with the assumption (8) on the initial data, that

$$
\|\omega\|^{2}+\|j\|^{2}+\int_{0}^{t}\left(\left\|\omega_{x}\right\|^{2}+\left\|\omega_{y}\right\|^{2}+\left\|j_{x}\right\|^{2}+\left\|j_{y}\right\|^{2}\right) \mathrm{d} s \leq 1
$$

Thus, the proof of Lemma 2.2 is completed.

Now, we turn to deduce the higher order estimates about the solution.

Lemma 2.3 If $(u, b)$ is the solution of (1)-(4), then

$$
\|\nabla \omega\|^{2}+\|\nabla j\|^{2}+\int_{0}^{t}\left(\left\|\nabla \omega_{x}\right\|^{2}+\left\|\nabla \omega_{y}\right\|^{2}+\left\|\nabla j_{x}\right\|^{2}+\left\|\nabla j_{y}\right\|^{2}\right) \mathrm{d} s \leq C .
$$

Proof Multiplying (6) and (7) by $\Delta \omega$ and $\Delta j$, respectively, then integrating the resultant equations by parts, after adding the two equalities together, we finally obtain

$$
\begin{aligned}
\frac{1}{2} & \frac{\mathrm{d}}{\mathrm{d} t}\left(\|\nabla \omega\|^{2}+\|\nabla j\|^{2}\right)+\left(\left\|\nabla \omega_{x}\right\|^{2}+\left\|\nabla \omega_{y}\right\|^{2}+\left\|\nabla j_{x}\right\|^{2}+\left\|\nabla j_{y}\right\|^{2}\right) \\
= & \int[u \cdot \nabla \omega \cdot \Delta \omega+u \cdot \nabla j \cdot \Delta j-\omega \cdot \nabla \omega \cdot \Delta \omega+j \cdot \nabla b \cdot \Delta \omega-b \cdot \nabla j \cdot \Delta \omega \\
& \left.\quad-b \cdot \nabla \omega \cdot \Delta j-\varepsilon_{i j k}\left(\partial_{j} b_{l} \partial_{l} u_{k}-\partial_{j} u_{l} \partial_{l} b_{k}\right) \Delta j_{i}\right] \mathrm{d} x \\
= & M+N+P+Q+R+S .
\end{aligned}
$$


Now, we turn to bound each term on the right-hand side of (11). Similar as the proof of Lemma 2.2, keeping in mind Lemma 2.1 and the divergence-free property of $u$ and $b$, we deduce

$$
\begin{aligned}
M & =\int u \cdot \nabla \omega \cdot \Delta \omega \mathrm{d} x=\int u_{i} \partial_{i} \omega_{j} \partial_{k k}^{2} \omega_{j} \mathrm{~d} x=-\int \partial_{k} u_{i} \partial_{i} \omega_{j} \partial_{k} \omega_{j} \mathrm{~d} x \\
& =-\int \partial_{x} u_{i} \partial_{i} \omega_{j} \partial_{x} \omega_{j} \mathrm{~d} x-\int \partial_{y} u_{i} \partial_{i} \omega_{j} \partial_{y} \omega_{j} \mathrm{~d} x-\int \partial_{z} u_{i} \partial_{i} \omega_{j} \partial_{z} \omega_{j} \mathrm{~d} x \\
& =M_{1}+M_{2}+M_{3} .
\end{aligned}
$$

We estimate each term as follows:

$$
\begin{aligned}
M_{1} & =-\int \partial_{x} u_{i} \partial_{i} \omega_{j} \partial_{x} \omega_{j} \mathrm{~d} x \leq C\left\|u_{x}\right\|^{\frac{1}{2}}\|\nabla \omega\|^{\frac{1}{2}}\|\nabla \omega\|^{\frac{1}{2}}\left\|\nabla \omega_{x}\right\|^{\frac{1}{2}}\left\|\nabla \omega_{y}\right\|^{\frac{1}{2}}\left\|\partial_{x} u_{z}\right\|^{\frac{1}{2}} \\
& \leq \frac{1}{62}\left\|\nabla \omega_{x}\right\|^{2}+\frac{1}{40}\left\|\nabla \omega_{y}\right\|^{2}+C\left\|u_{x}\right\|\left\|\omega_{x}\right\|\|\nabla \omega\|^{2} \\
& \leq \frac{1}{62}\left\|\nabla \omega_{x}\right\|^{2}+\frac{1}{40}\left\|\nabla \omega_{y}\right\|^{2}+C\left(\left\|u_{x}\right\|^{2}+\left\|\omega_{x}\right\|^{2}\right)\|\nabla \omega\|^{2} .
\end{aligned}
$$

Similarly,

$$
\begin{aligned}
M_{2} & =-\int \partial_{y} u_{i} \partial_{i} \omega_{j} \partial_{y} \omega_{j} \mathrm{~d} x \\
& \leq C\left\|u_{y}\right\|^{\frac{1}{2}}\|\nabla \omega\|^{\frac{1}{2}}\|\nabla \omega\|^{\frac{1}{2}}\left\|\nabla \omega_{x}\right\|^{\frac{1}{2}}\left\|\nabla \omega_{y}\right\|^{\frac{1}{2}}\left\|\partial_{y} u_{z}\right\|^{\frac{1}{2}} \\
& \leq \frac{1}{62}\left\|\nabla \omega_{x}\right\|^{2}+\frac{1}{40}\left\|\nabla \omega_{y}\right\|^{2}+C\left(\left\|u_{y}\right\|^{2}+\left\|\omega_{y}\right\|^{2}\right)\|\nabla \omega\|^{2} .
\end{aligned}
$$

Now, we turn to bound $M_{3}$,

$$
\begin{aligned}
M_{3} & =-\int \partial_{z} u_{i} \partial_{i} \omega_{j} \partial_{z} \omega_{j} \mathrm{~d} x \\
& =-\int \partial_{z} u_{1} \partial_{x} \omega_{j} \partial_{z} \omega_{j} \mathrm{~d} x-\int \partial_{z} u_{2} \partial_{y} \omega_{j} \partial_{z} \omega_{j} \mathrm{~d} x-\int \partial_{z} u_{3} \partial_{z} \omega_{j} \partial_{z} \omega_{j} \mathrm{~d} x \\
& =M_{31}+M_{32}+M_{33} .
\end{aligned}
$$

Similarly, we can deduce that

$$
\begin{aligned}
M_{31} & =-\int \partial_{z} u_{1} \partial_{x} \omega_{j} \partial_{z} \omega_{j} \mathrm{~d} x \\
& \leq C\|\omega\|^{\frac{1}{2}}\left\|\omega_{x}\right\|^{\frac{1}{2}}\|\nabla \omega\|^{\frac{1}{2}}\left\|\omega_{x}\right\|^{\frac{1}{2}}\left\|\nabla \omega_{x}\right\|^{\frac{1}{2}}\left\|\nabla \omega_{y}\right\|^{\frac{1}{2}} \\
& \leq \frac{1}{62}\left\|\nabla \omega_{x}\right\|^{2}+\frac{1}{40}\left\|\nabla \omega_{y}\right\|^{2}+C\left(\|\omega\|^{2}+\left\|\omega_{x}\right\|^{2}\right)\|\nabla \omega\|^{2}, \\
M_{32} & =-\int \partial_{z} u_{2} \partial_{y} \omega_{j} \partial_{z} \omega_{j} \mathrm{~d} x \\
& \leq C\|\omega\|^{\frac{1}{2}}\left\|\omega_{y}\right\|^{\frac{1}{2}}\|\nabla \omega\|^{\frac{1}{2}}\left\|\omega_{x}\right\|^{\frac{1}{2}}\left\|\nabla \omega_{y}\right\|^{\frac{1}{2}}\left\|\nabla \omega_{y}\right\|^{\frac{1}{2}} \\
& \leq \frac{1}{40}\left\|\nabla \omega_{y}\right\|^{2}+C\left(\|\omega\|^{2}+\left\|\omega_{x}\right\|^{2}\right)\|\nabla \omega\|^{2},
\end{aligned}
$$




$$
\begin{aligned}
M_{33}= & -\int \partial_{z} u_{3} \partial_{z} \omega_{j} \partial_{z} \omega_{j} \mathrm{~d} x=\int\left(\partial_{x} u_{1}+\partial_{y} u_{2}\right) \partial_{z} \omega_{j} \partial_{z} \omega_{j} \mathrm{~d} x \\
\leq & C\left\|u_{x}\right\|^{\frac{1}{2}}\|\nabla \omega\|^{\frac{1}{2}}\|\nabla \omega\|^{\frac{1}{2}}\left\|\partial_{x} u_{z}\right\|^{\frac{1}{2}}\left\|\nabla \omega_{x}\right\|^{\frac{1}{2}}\left\|\nabla \omega_{y}\right\|^{\frac{1}{2}} \\
& +C\left\|u_{y}\right\|^{\frac{1}{2}}\|\nabla \omega\|^{\frac{1}{2}}\|\nabla \omega\|^{\frac{1}{2}}\left\|\partial_{y} u_{z}\right\|^{\frac{1}{2}}\left\|\nabla \omega_{x}\right\|^{\frac{1}{2}}\left\|\nabla \omega_{y}\right\|^{\frac{1}{2}} \\
\leq & \frac{1}{62}\left\|\nabla \omega_{x}\right\|^{2}+\frac{1}{40}\left\|\nabla \omega_{y}\right\|^{2}+C\left(\left\|u_{x}\right\|^{2}+\left\|u_{y}\right\|^{2}+\left\|\omega_{x}\right\|^{2}+\left\|\omega_{y}\right\|^{2}\right)\|\nabla \omega\|^{2} .
\end{aligned}
$$

As for $N$, integrating by parts, we deduce that

$$
\begin{aligned}
N & =\int u \cdot \nabla j \cdot \Delta j \mathrm{~d} x=\int u_{i} \partial_{i} j_{j} \partial_{k k}^{2} j_{j} \mathrm{~d} x=-\int \partial_{k} u_{i} \partial_{i} j_{j} \partial_{k} j_{j} \mathrm{~d} x \\
& =-\int \partial_{x} u_{i} \partial_{i} j_{j} \partial_{x} j_{j} \mathrm{~d} x-\int \partial_{y} u_{i} \partial_{i} j_{j} \partial_{y} j_{j} \mathrm{~d} x-\int \partial_{z} u_{i} \partial_{i} j_{j} \partial_{z} j_{j} \mathrm{~d} x \\
& =N_{1}+N_{2}+N_{3} .
\end{aligned}
$$

Similarly, we have

$$
\begin{aligned}
N_{1} & =-\int \partial_{x} u_{i} \partial_{i} j_{j} \partial_{x} j_{j} \mathrm{~d} x \\
& \leq C\left\|u_{x}\right\|^{\frac{1}{2}}\|\nabla j\|^{\frac{1}{2}}\left\|j_{x}\right\|^{\frac{1}{2}}\left\|\omega_{x}\right\|^{\frac{1}{2}}\left\|\nabla j_{x}\right\|^{\frac{1}{2}}\left\|\nabla j_{y}\right\|^{\frac{1}{2}} \\
& \leq \frac{1}{50}\left\|\nabla j_{x}\right\|^{2}+\frac{1}{48}\left\|\nabla j_{y}\right\|^{2}+C\left(\left\|u_{x}\right\|^{2}+\left\|\omega_{x}\right\|^{2}\right)\|\nabla j\|^{2}, \\
N_{2} & =-\int \partial_{y} u_{i} \partial_{i} j_{j} \partial_{y} j_{j} \mathrm{~d} x \\
& \leq C\left\|u_{y}\right\|^{\frac{1}{2}}\|\nabla j\|^{\frac{1}{2}}\left\|j_{y}\right\|^{\frac{1}{2}}\left\|\omega_{y}\right\|^{\frac{1}{2}}\left\|\nabla j_{x}\right\|^{\frac{1}{2}}\left\|\nabla j_{y}\right\|^{\frac{1}{2}} \\
& \leq \frac{1}{50}\left\|\nabla j_{x}\right\|^{2}+\frac{1}{48}\left\|\nabla j_{y}\right\|^{2}+C\left(\left\|u_{y}\right\|^{2}+\left\|\omega_{y}\right\|^{2}\right)\|\nabla j\|^{2} .
\end{aligned}
$$

As for $N_{3}$, we obtain

$$
\begin{aligned}
N_{3} & =-\int \partial_{z} u_{i} \partial_{i} j_{j} \partial_{z} j_{j} \mathrm{~d} x \\
& =-\int \partial_{z} u_{1} \partial_{x} j_{j} \partial_{z} j_{j} \mathrm{~d} x-\int \partial_{z} u_{2} \partial_{y} j_{j} \partial_{z} j_{j} \mathrm{~d} x-\int \partial_{z} u_{3} \partial_{z} j_{j} \partial_{z} j_{j} \mathrm{~d} x \\
& =N_{31}+N_{32}+N_{33} .
\end{aligned}
$$

Thus, we have

$$
\begin{aligned}
N_{31} & =-\int \partial_{z} u_{1} \partial_{x} j_{j} \partial_{z} j_{j} \mathrm{~d} x \\
& \leq C\|\omega\|^{\frac{1}{2}}\left\|j_{x}\right\|^{\frac{1}{2}}\|\nabla j\|^{\frac{1}{2}}\left\|\omega_{x}\right\|^{\frac{1}{2}}\left\|\nabla j_{x}\right\|^{\frac{1}{2}}\left\|\nabla j_{y}\right\|^{\frac{1}{2}} \\
& \leq \frac{1}{50}\left\|\nabla j_{x}\right\|^{2}+\frac{1}{48}\left\|\nabla j_{y}\right\|^{2}+C\left(\|\omega\|^{2}+\left\|\omega_{x}\right\|^{2}\right)\|\nabla j\|^{2},
\end{aligned}
$$




$$
\begin{aligned}
N_{32}= & -\int \partial_{z} u_{2} \partial_{y} j_{j} \partial_{z} j_{j} \mathrm{~d} x \\
\leq & C\|\omega\|^{\frac{1}{2}}\left\|j_{y}\right\|^{\frac{1}{2}}\|\nabla j\|^{\frac{1}{2}}\left\|\omega_{x}\right\|^{\frac{1}{2}}\left\|\nabla j_{y}\right\|^{\frac{1}{2}}\left\|\nabla j_{y}\right\|^{\frac{1}{2}} \\
\leq & \frac{1}{48}\left\|\nabla j_{y}\right\|^{2}+C\left(\|\omega\|^{2}+\left\|\omega_{x}\right\|^{2}\right)\|\nabla j\|^{2}, \\
N_{33}= & -\int \partial_{z} u_{3} \partial_{z} j_{j} \partial_{z} j_{j} \mathrm{~d} x \\
\leq & C\left\|u_{x}\right\|^{\frac{1}{2}}\|\nabla j\|^{\frac{1}{2}}\|\nabla j\|^{\frac{1}{2}}\left\|\nabla j_{x}\right\|^{\frac{1}{2}}\left\|\nabla j_{y}\right\|^{\frac{1}{2}}\left\|\omega_{x}\right\|^{\frac{1}{2}} \\
& +C\left\|u_{y}\right\|^{\frac{1}{2}}\|\nabla j\|^{\frac{1}{2}}\|\nabla j\|^{\frac{1}{2}}\left\|\nabla j_{x}\right\|^{\frac{1}{2}}\left\|\nabla j_{y}\right\|^{\frac{1}{2}}\left\|\omega_{y}\right\|^{\frac{1}{2}} \\
\leq & \frac{1}{50}\left\|\nabla j_{x}\right\|^{2}+\frac{1}{48}\left\|\nabla j_{y}\right\|^{2}+C\left(\left\|u_{x}\right\|^{2}+\left\|u_{y}\right\|^{2}+\left\|\omega_{x}\right\|^{2}+\left\|\omega_{y}\right\|^{2}\right)\|\nabla j\|^{2} .
\end{aligned}
$$

We now turn to bound $P$,

$$
\begin{aligned}
P & =-\int \omega \cdot \nabla \omega \cdot \Delta \omega \mathrm{d} x=-\int \omega_{i} \partial_{i} u_{j} \partial_{k k}^{2} \omega_{j} \mathrm{~d} x \\
& =\int \partial_{k} \omega_{i} \partial_{i} u_{j} \partial_{k} \omega_{j} \mathrm{~d} x+\int \omega_{i} \partial_{k} \partial_{i} u_{j} \partial_{k} \omega_{j} \mathrm{~d} x=P_{1}+P_{2} .
\end{aligned}
$$

For $P_{1}$, we have

$$
\begin{aligned}
P_{1} & =\int \partial_{k} \omega_{i} \partial_{i} u_{j} \partial_{k} \omega_{j} \mathrm{~d} x \\
& =\int \partial_{x} \omega_{i} \partial_{i} u_{j} \partial_{x} \omega_{j} \mathrm{~d} x+\int \partial_{y} \omega_{i} \partial_{i} u_{j} \partial_{y} \omega_{j} \mathrm{~d} x+\int \partial_{z} \omega_{i} \partial_{i} u_{j} \partial_{z} \omega_{j} \mathrm{~d} x \\
& =P_{11}+P_{12}+P_{13}, \\
P_{11} & =\int \partial_{x} \omega_{i} \partial_{i} u_{j} \partial_{x} \omega_{j} \mathrm{~d} x \\
& \leq C\left\|\omega_{x}\right\|^{\frac{1}{2}}\|\omega\|^{\frac{1}{2}}\left\|\omega_{x}\right\|^{\frac{1}{2}}\left\|\omega_{x}\right\|^{\frac{1}{2}}\left\|\nabla \omega_{x}\right\|^{\frac{1}{2}}\left\|\nabla \omega_{x}\right\|^{\frac{1}{2}} \\
& \leq \frac{1}{62}\left\|\nabla \omega_{x}\right\|^{2}+C\left(\|\omega\|^{2}+\left\|\omega_{x}\right\|^{2}\right)\|\nabla \omega\|^{2}, \\
P_{12} & =\int \partial_{y} \omega_{i} \partial_{i} u_{j} \partial_{y} \omega_{j} \mathrm{~d} x \\
& \leq C\left\|\omega_{y}\right\|^{\frac{1}{2}}\|\omega\|^{\frac{1}{2}}\left\|\omega_{y}\right\|^{\frac{1}{2}}\left\|\omega_{x}\right\|^{\frac{1}{2}}\left\|\nabla \omega_{y}\right\|^{\frac{1}{2}}\left\|\nabla \omega_{y}\right\|^{\frac{1}{2}} \\
& \leq \frac{1}{40}\left\|\nabla \omega_{y}\right\|^{2}+C\left(\|\omega\|^{2}+\left\|\omega_{x}\right\|^{2}\right)\|\nabla \omega\|^{2} .
\end{aligned}
$$

For $P_{13}$, we see that

$$
\begin{aligned}
P_{13} & =\int \partial_{z} \omega_{i} \partial_{i} u_{j} \partial_{z} \omega_{j} \mathrm{~d} x \\
& =\int \partial_{z} \omega_{1} \partial_{x} u_{j} \partial_{z} \omega_{j} \mathrm{~d} x+\int \partial_{z} \omega_{2} \partial_{y} u_{j} \partial_{z} \omega_{j} \mathrm{~d} x+\int \partial_{z} \omega_{3} \partial_{z} u_{j} \partial_{z} \omega_{j} \mathrm{~d} x \\
& =P_{13}^{1}+P_{13}^{2}+P_{13}^{3} .
\end{aligned}
$$


Thus, we can bound $P_{13}$ as follows:

$$
\begin{aligned}
P_{13}^{1}= & \int \partial_{z} \omega_{1} \partial_{x} u_{j} \partial_{z} \omega_{j} \mathrm{~d} x \leq C\|\nabla \omega\|^{\frac{1}{2}}\left\|u_{x}\right\|^{\frac{1}{2}}\|\nabla \omega\|^{\frac{1}{2}}\left\|\nabla \omega_{x}\right\|^{\frac{1}{2}}\left\|\nabla \omega_{y}\right\|^{\frac{1}{2}}\left\|\omega_{x}\right\|^{\frac{1}{2}} \\
\leq & \frac{1}{62}\left\|\nabla \omega_{x}\right\|^{2}+\frac{1}{40}\left\|\nabla \omega_{y}\right\|^{2}+C\left(\left\|u_{x}\right\|^{2}+\left\|\omega_{x}\right\|^{2}\right)\|\nabla \omega\|^{2}, \\
P_{13}^{2}= & \int \partial_{z} \omega_{2} \partial_{y} u_{j} \partial_{z} \omega_{j} \mathrm{~d} x \leq C\|\nabla \omega\|^{\frac{1}{2}}\left\|u_{y}\right\|^{\frac{1}{2}}\|\nabla \omega\|^{\frac{1}{2}}\left\|\nabla \omega_{x}\right\|^{\frac{1}{2}}\left\|\nabla \omega_{y}\right\|^{\frac{1}{2}}\left\|\omega_{y}\right\|^{\frac{1}{2}} \\
\leq & \frac{1}{62}\left\|\nabla \omega_{x}\right\|^{2}+\frac{1}{40}\left\|\nabla \omega_{y}\right\|^{2}+C\left(\left\|u_{y}\right\|^{2}+\left\|\omega_{y}\right\|^{2}\right)\|\nabla \omega\|^{2}, \\
P_{13}^{2}= & \int \partial_{z} \omega_{3} \partial_{z} u_{j} \partial_{z} \omega_{j} \mathrm{~d} x \\
= & \int \partial_{z}\left(\partial_{x} u_{2}-\partial_{y} u_{1}\right) \partial_{z} u_{j} \partial_{z} \omega_{j} \mathrm{~d} x \\
\leq & C\left\|\omega_{x}\right\|^{\frac{1}{2}}\|\omega\|^{\frac{1}{2}}\|\nabla \omega\|^{\frac{1}{2}}\left\|\nabla \omega_{x}\right\|^{\frac{1}{2}}\left\|\omega_{x}\right\|^{\frac{1}{2}}\left\|\nabla \omega_{y}\right\|^{\frac{1}{2}} \\
& +C\left\|\omega_{y}\right\|^{\frac{1}{2}}\|\omega\|^{\frac{1}{2}}\|\nabla \omega\|^{\frac{1}{2}}\left\|\nabla \omega_{y}\right\|^{\frac{1}{2}}\left\|\omega_{y}\right\|^{\frac{1}{2}}\left\|\nabla \omega_{x}\right\|^{\frac{1}{2}} \\
\leq & \frac{1}{62}\left\|\nabla \omega_{x}\right\|^{2}+\frac{1}{40}\left\|\nabla \omega_{y}\right\|^{2}+C\left(\|\omega\|^{2}+\left\|\omega_{x}\right\|^{2}+\left\|\omega_{y}\right\|^{2}\right)\|\nabla \omega\|^{2} .
\end{aligned}
$$

Now, we turn to $P_{2}$,

$$
\begin{aligned}
P_{2} & =\int \omega_{i} \partial_{k} \partial_{i} u_{j} \partial_{k} \omega_{j} \mathrm{~d} x \\
& =\int \omega_{1} \partial_{k} \partial_{x} u_{j} \partial_{k} \omega_{j} \mathrm{~d} x+\int \omega_{2} \partial_{k} \partial_{y} u_{j} \partial_{k} \omega_{j} \mathrm{~d} x+\int \omega_{3} \partial_{k} \partial_{z} u_{j} \partial_{k} \omega_{j} \mathrm{~d} x \\
& =P_{21}+P_{22}+P_{23} .
\end{aligned}
$$

Similarly,

$$
\begin{aligned}
P_{21}= & \int \omega_{1} \partial_{k} \partial_{x} u_{j} \partial_{k} \omega_{j} \mathrm{~d} x \\
\leq & C\|\omega\|^{\frac{1}{2}}\left\|\omega_{x}\right\|^{\frac{1}{2}}\|\nabla \omega\|^{\frac{1}{2}}\left\|\omega_{x}\right\|^{\frac{1}{2}}\left\|\nabla \omega_{y}\right\|^{\frac{1}{2}}\left\|\nabla \omega_{x}\right\|^{\frac{1}{2}} \\
\leq & \frac{1}{62}\left\|\nabla \omega_{x}\right\|^{2}+\frac{1}{40}\left\|\nabla \omega_{y}\right\|^{2}+C\left(\|\omega\|^{2}+\left\|\omega_{x}\right\|^{2}\right)\|\nabla \omega\|^{2}, \\
P_{22}= & \int \omega_{2} \partial_{k} \partial_{y} u_{j} \partial_{k} \omega_{j} \mathrm{~d} x \\
\leq & C\|\omega\|^{\frac{1}{2}}\left\|\omega_{y}\right\|^{\frac{1}{2}}\|\nabla \omega\|^{\frac{1}{2}}\left\|\omega_{x}\right\|^{\frac{1}{2}}\left\|\nabla \omega_{y}\right\|^{\frac{1}{2}}\left\|\nabla \omega_{y}\right\|^{\frac{1}{2}} \\
\leq & \frac{1}{40}\left\|\nabla \omega_{y}\right\|^{2}+C\left(\|\omega\|^{2}+\left\|\omega_{x}\right\|^{2}\right)\|\nabla \omega\|^{2}, \\
P_{23}= & \int \omega_{3} \partial_{k} \partial_{z} u_{j} \partial_{k} \omega_{j} \mathrm{~d} x \\
\leq & C\left\|u_{x}\right\|^{\frac{1}{2}}\|\nabla \omega\|^{\frac{1}{2}}\|\nabla \omega\|^{\frac{1}{2}}\left\|\nabla \omega_{x}\right\|^{\frac{1}{2}}\left\|\nabla \omega_{y}\right\|^{\frac{1}{2}}\left\|\omega_{x}\right\|^{\frac{1}{2}} \\
& +C\left\|u_{y}\right\|^{\frac{1}{2}}\|\nabla \omega\|^{\frac{1}{2}}\|\nabla \omega\|^{\frac{1}{2}}\left\|\nabla \omega_{x}\right\|^{\frac{1}{2}}\left\|\nabla \omega_{y}\right\|^{\frac{1}{2}}\left\|\omega_{y}\right\|^{\frac{1}{2}} \\
\leq & \frac{1}{62}\left\|\nabla \omega_{x}\right\|^{2}+\frac{1}{40}\left\|\nabla \omega_{y}\right\|^{2}+C\left(\left\|u_{x}\right\|^{2}+\left\|u_{y}\right\|^{2}+\left\|\omega_{x}\right\|^{2}+\left\|\omega_{y}\right\|^{2}\right)\|\nabla \omega\|^{2} .
\end{aligned}
$$


Si and Wang Journal of Inequalities and Applications 2013, 2013:345

Page 11 of 18

http://www.journalofinequalitiesandapplications.com/content/2013/1/345

To bound $Q$, we see that

$$
\begin{aligned}
Q & =\int j \cdot \nabla b \cdot \Delta \omega \mathrm{d} x=\int j_{i} \partial_{i} b_{j} \partial_{k k}^{2} \omega_{j} \mathrm{~d} x \\
& =-\int \partial_{k} j_{i} \partial_{i} b_{j} \partial_{k} \omega_{j} \mathrm{~d} x-\int j_{i} \partial_{k} \partial_{i} b_{j} \partial_{k} \omega_{j} \mathrm{~d} x=Q_{1}+Q_{2}, \\
Q_{1} & =-\int \partial_{x} j_{i} \partial_{i} b_{j} \partial_{x} \omega_{j} \mathrm{~d} x-\int \partial_{y} j_{i} \partial_{i} b_{j} \partial_{y} \omega_{j} \mathrm{~d} x-\int \partial_{z} j_{i} \partial_{i} b_{j} \partial_{z} \omega_{j} \mathrm{~d} x \\
& =Q_{11}+Q_{12}+Q_{13}, \\
Q_{11} & =-\int \partial_{x} j_{i} \partial_{i} b_{j} \partial_{x} \omega_{j} \mathrm{~d} x \leq C\left\|j_{x}\right\|^{\frac{1}{2}}\|j\|^{\frac{1}{2}}\left\|\omega_{x}\right\|^{\frac{1}{2}}\left\|\nabla j_{x}\right\|^{\frac{1}{2}}\|\nabla j\|^{\frac{1}{2}}\left\|\nabla \omega_{x}\right\|^{\frac{1}{2}} \\
& \leq \frac{1}{62}\left\|\nabla \omega_{x}\right\|^{2}+\frac{1}{50}\left\|\nabla j_{x}\right\|^{2}+C\left(\|j\|^{2}+\left\|j_{x}\right\|^{2}\right)\left(\|\nabla \omega\|^{2}+\|\nabla j\|^{2}\right), \\
Q_{12} & =-\int \partial_{y} j_{i} \partial_{i} b_{j} \partial_{y} \omega_{j} \mathrm{~d} x \leq C\left\|j_{y}\right\|^{\frac{1}{2}}\|j\|^{\frac{1}{2}}\left\|\omega_{x}\right\|^{\frac{1}{2}}\left\|\nabla j_{y}\right\|^{\frac{1}{2}}\left\|j_{x}\right\|^{\frac{1}{2}}\left\|\nabla \omega_{x}\right\|^{\frac{1}{2}} \\
& \leq \frac{1}{62}\left\|\nabla \omega_{x}\right\|^{2}+\frac{1}{48}\left\|\nabla j_{y}\right\|^{2}+C\left(\|j\|^{2}+\left\|j_{y}\right\|^{2}\right)\left(\|\nabla \omega\|^{2}+\|\nabla j\|^{2}\right) .
\end{aligned}
$$

As for $Q_{13}$, we see that

$$
\begin{aligned}
Q_{13} & =-\int \partial_{z} j_{i} \partial_{i} b_{j} \partial_{z} \omega_{j} \mathrm{~d} x \\
& =-\int \partial_{z} j_{1} \partial_{x} b_{j} \partial_{z} \omega_{j} \mathrm{~d} x-\int \partial_{z} j_{2} \partial_{y} b_{j} \partial_{z} \omega_{j} \mathrm{~d} x-\int \partial_{z} j_{3} \partial_{z} b_{j} \partial_{z} \omega_{j} \mathrm{~d} x \\
& =Q_{13}^{1}+Q_{13}^{2}+Q_{13}^{3} .
\end{aligned}
$$

Thus, we can deduce that

$$
\begin{aligned}
Q_{13}^{1}= & -\int \partial_{z} j_{1} \partial_{x} b_{j} \partial_{z} \omega_{j} \mathrm{~d} x \\
\leq & C\|\nabla j\|^{\frac{1}{2}}\left\|b_{x}\right\|^{\frac{1}{2}}\|\nabla \omega\|^{\frac{1}{2}}\left\|\nabla j_{x}\right\|^{\frac{1}{2}}\left\|j_{x}\right\|^{\frac{1}{2}}\left\|\nabla \omega_{y}\right\|^{\frac{1}{2}} \\
& \leq \frac{1}{40}\left\|\nabla \omega_{y}\right\|^{2}+\frac{1}{50}\left\|\nabla j_{x}\right\|^{2}+C\left(\left\|b_{x}\right\|^{2}+\left\|j_{x}\right\|^{2}\right)\left(\|\nabla \omega\|^{2}+\|\nabla j\|^{2}\right), \\
Q_{13}^{2}= & -\int \partial_{z} j_{2} \partial_{y} b_{j} \partial_{z} \omega_{j} \mathrm{~d} x \\
\leq & C\|\nabla j\|^{\frac{1}{2}}\left\|b_{x}\right\|^{\frac{1}{2}}\|\nabla \omega\|^{\frac{1}{2}}\left\|\nabla j_{x}\right\|^{\frac{1}{2}}\left\|j_{y}\right\|^{\frac{1}{2}}\left\|\nabla \omega_{y}\right\|^{\frac{1}{2}} \\
\leq & \frac{1}{40}\left\|\nabla \omega_{y}\right\|^{2}+\frac{1}{50}\left\|\nabla j_{x}\right\|^{2}+C\left(\left\|b_{y}\right\|^{2}+\left\|j_{y}\right\|^{2}\right)\left(\|\nabla \omega\|^{2}+\|\nabla j\|^{2}\right), \\
Q_{13}^{3}= & -\int \partial_{z} j_{3} \partial_{z} b_{j} \partial_{z} \omega_{j} \mathrm{~d} x=-\int \partial_{z}\left(\partial_{x} b_{2}-\partial_{y} b_{1}\right) \partial_{z} b_{j} \partial_{z} \omega_{j} \mathrm{~d} x \\
\leq & C\left\|j_{x}\right\|^{\frac{1}{2}}\|j\|^{\frac{1}{2}}\|\nabla \omega\|^{\frac{1}{2}}\left\|\nabla j_{x}\right\|^{\frac{1}{2}}\left\|j_{y}\right\|^{\frac{1}{2}}\left\|\nabla \omega_{x}\right\|^{\frac{1}{2}} \\
& +C\left\|j_{y}\right\|^{\frac{1}{2}}\|j\|^{\frac{1}{2}}\|\nabla \omega\|^{\frac{1}{2}}\left\|\nabla j_{y}\right\|^{\frac{1}{2}}\left\|j_{y}\right\|^{\frac{1}{2}}\left\|\nabla \omega_{x}\right\|^{\frac{1}{2}} \\
\leq & \frac{1}{62}\left\|\nabla \omega_{x}\right\|^{2}+\frac{1}{50}\left\|\nabla j_{x}\right\|^{2}+\frac{1}{48}\left\|\nabla j_{y}\right\|^{2}+C\left(\|j\|^{2}+\left\|j_{x}\right\|^{2}+\left\|j_{y}\right\|^{2}\right)\left(\|\nabla \omega\|^{2}+\|\nabla j\|^{2}\right) .
\end{aligned}
$$


Now, we turn to $Q_{2}$,

$$
\begin{aligned}
Q_{2} & =-\int b \cdot \nabla j \cdot \Delta \omega \mathrm{d} x=-\int j_{i} \partial_{k} \partial_{i} b_{j} \partial_{k} \omega_{j} \mathrm{~d} x \\
& =-\int j_{1} \partial_{k} \partial_{x} b_{j} \partial_{k} \omega_{j} \mathrm{~d} x-\int j_{2} \partial_{k} \partial_{y} b_{j} \partial_{k} \omega_{j} \mathrm{~d} x-\int j_{3} \partial_{k} \partial_{z} b_{j} \partial_{k} \omega_{j} \mathrm{~d} x \\
& =Q_{21}+Q_{22}+Q_{23} .
\end{aligned}
$$

We deduce that

$$
\begin{aligned}
Q_{21}= & -\int j_{1} \partial_{k} \partial_{x} b_{j} \partial_{k} \omega_{j} \mathrm{~d} x \\
\leq & C\|j\|^{\frac{1}{2}}\left\|j_{x}\right\|^{\frac{1}{2}}\|\nabla \omega\|^{\frac{1}{2}}\left\|\nabla \omega_{x}\right\|^{\frac{1}{2}}\left\|j_{y}\right\|^{\frac{1}{2}}\left\|\nabla j_{x}\right\|^{\frac{1}{2}} \\
\leq & \frac{1}{62}\left\|\nabla \omega_{x}\right\|^{2}+\frac{1}{50}\left\|\nabla j_{x}\right\|^{2}+C\left(\|j\|^{2}+\left\|j_{x}\right\|^{2}\right)\left(\|\nabla \omega\|^{2}+\|\nabla j\|^{2}\right), \\
Q_{22}= & -\int j_{2} \partial_{k} \partial_{y} b_{j} \partial_{k} \omega_{j} \mathrm{~d} x \\
\leq & C\|j\|^{\frac{1}{2}}\left\|j_{y}\right\|^{\frac{1}{2}}\|\nabla \omega\|^{\frac{1}{2}}\left\|\nabla \omega_{x}\right\|^{\frac{1}{2}}\left\|j_{y}\right\|^{\frac{1}{2}}\left\|\nabla j_{y}\right\|^{\frac{1}{2}} \\
\leq & \frac{1}{62}\left\|\nabla \omega_{x}\right\|^{2}+\frac{1}{48}\left\|\nabla j_{y}\right\|^{2}+C\left(\|j\|^{2}+\left\|j_{y}\right\|^{2}\right)\left(\|\nabla \omega\|^{2}+\|\nabla j\|^{2}\right), \\
Q_{23}= & -\int j_{3} \partial_{k} \partial_{z} b_{j} \partial_{k} \omega_{j} \mathrm{~d} x \\
\leq & C\left\|b_{x}\right\|^{\frac{1}{2}}\|\nabla j\|^{\frac{1}{2}}\|\nabla \omega\|^{\frac{1}{2}}\left\|\nabla \omega_{x}\right\|^{\frac{1}{2}}\left\|\nabla j_{y}\right\|^{\frac{1}{2}}\left\|j_{x}\right\|^{\frac{1}{2}} \\
& +C\left\|b_{y}\right\|^{\frac{1}{2}}\|\nabla j\|^{\frac{1}{2}}\|\nabla \omega\|^{\frac{1}{2}}\left\|\nabla \omega_{x}\right\|^{\frac{1}{2}}\left\|\nabla j_{y}\right\|^{\frac{1}{2}}\left\|j_{y}\right\|^{\frac{1}{2}} \\
\leq & \frac{1}{62}\left\|\nabla \omega_{x}\right\|^{2}+\frac{1}{48}\left\|\nabla j_{y}\right\|^{2}+C\left(\left\|b_{x}\right\|^{2}+\left\|j_{x}\right\|^{2}+\left\|b_{y}\right\|^{2}+\left\|j_{y}\right\|^{2}\right)\left(\|\nabla \omega\|^{2}+\|\nabla j\|^{2}\right) .
\end{aligned}
$$

Here we start to estimate $R$ as follows:

$$
\begin{aligned}
R & =-\int b \cdot \nabla j \cdot \Delta \omega \mathrm{d} x-\int b \cdot \nabla \omega \cdot \Delta j \mathrm{~d} x \\
& =-\int b_{i} \partial_{i} j_{j} \partial_{k k}^{2} \omega_{j} \mathrm{~d} x-\int b_{i} \partial_{i} \omega_{j} \partial_{k k}^{2} j_{j} \mathrm{~d} x \\
& =\int \partial_{k} b_{i} \partial_{i} j_{j} \partial_{k} \omega_{j} \mathrm{~d} x+\int \partial_{k} b_{i} \partial_{i} \omega_{j} \partial_{k} j_{j} \mathrm{~d} x=R_{1}+R_{2} .
\end{aligned}
$$

For $R_{1}$, we have

$$
\begin{aligned}
R_{1} & =\int \partial_{k} b_{i} \partial_{i} j_{j} \partial_{k} \omega_{j} \mathrm{~d} x \\
& =\int \partial_{x} b_{i} \partial_{i} j_{j} \partial_{x} \omega_{j} \mathrm{~d} x+\int \partial_{y} b_{i} \partial_{i} j_{j} \partial_{y} \omega_{j} \mathrm{~d} x+\int \partial_{z} b_{i} \partial_{i} j_{j} \partial_{z} \omega_{j} \mathrm{~d} x \\
& =R_{11}+R_{12}+R_{13} .
\end{aligned}
$$


We deduce that

$$
\begin{aligned}
R_{11} & =\int \partial_{x} b_{i} \partial_{i} j_{j} \partial_{x} \omega_{j} \mathrm{~d} x \\
& \leq C\left\|b_{x}\right\|^{\frac{1}{2}}\|\nabla j\|^{\frac{1}{2}}\left\|\omega_{x}\right\|^{\frac{1}{2}}\left\|\nabla j_{x}\right\|^{\frac{1}{2}}\left\|\nabla \omega_{x}\right\|^{\frac{1}{2}}\left\|j_{x}\right\|^{\frac{1}{2}} \\
& \leq \frac{1}{62}\left\|\nabla \omega_{x}\right\|^{2}+\frac{1}{50}\left\|\nabla j_{x}\right\|^{2}+C\left(\left\|b_{x}\right\|^{2}+\left\|\omega_{x}\right\|^{2}\right)\|\nabla j\|^{2}, \\
R_{12} & =\int \partial_{y} b_{i} \partial_{i} j_{j} \partial_{y} \omega_{j} \mathrm{~d} x \\
& \leq C\left\|b_{y}\right\|^{\frac{1}{2}}\|\nabla j\|^{\frac{1}{2}}\left\|\omega_{y}\right\|^{\frac{1}{2}}\left\|\nabla j_{x}\right\|^{\frac{1}{2}}\left\|\nabla \omega_{y}\right\|^{\frac{1}{2}}\left\|j_{y}\right\|^{\frac{1}{2}} \\
& \leq \frac{1}{50}\left\|\nabla j_{x}\right\|^{2}+\frac{1}{40}\left\|\nabla \omega_{y}\right\|^{2}+C\left(\left\|b_{y}\right\|^{2}+\left\|\omega_{y}\right\|^{2}\right)\|\nabla j\|^{2} .
\end{aligned}
$$

For $R_{13}$, we have

$$
\begin{aligned}
R_{13} & =\int \partial_{z} b_{i} \partial_{i} j_{j} \partial_{z} \omega_{j} \mathrm{~d} x \\
& =\int \partial_{z} b_{1} \partial_{x} j_{j} \partial_{z} \omega_{j} \mathrm{~d} x+\int \partial_{z} b_{2} \partial_{y} j_{j} \partial_{z} \omega_{j} \mathrm{~d} x+\int \partial_{z} b_{3} \partial_{z} j_{j} \partial_{z} \omega_{j} \mathrm{~d} x=R_{13}^{1}+R_{13}^{2}+R_{13}^{3} .
\end{aligned}
$$

Similarly,

$$
\begin{aligned}
R_{13}^{1}= & \int \partial_{z} b_{1} \partial_{x} j_{j} \partial_{z} \omega_{j} \mathrm{~d} x \\
\leq & C\|j\|^{\frac{1}{2}}\left\|j_{x}\right\|^{\frac{1}{2}}\|\nabla \omega\|^{\frac{1}{2}}\left\|\nabla \omega_{x}\right\|^{\frac{1}{2}}\left\|\nabla j_{x}\right\|^{\frac{1}{2}}\left\|j_{y}\right\|^{\frac{1}{2}} \\
\leq & \frac{1}{62}\left\|\nabla \omega_{x}\right\|^{2}+\frac{1}{50}\left\|\nabla j_{x}\right\|^{2}+C\left(\|j\|^{2}+\left\|j_{x}\right\|^{2}\right)\left(\|\nabla \omega\|^{2}+\|\nabla j\|^{2}\right), \\
R_{13}^{2}= & \int \partial_{z} b_{2} \partial_{y} j_{j} \partial_{z} \omega_{j} \mathrm{~d} x \\
\leq & C\|j\| \frac{1}{2}\left\|j_{y}\right\|^{\frac{1}{2}}\|\nabla \omega\|^{\frac{1}{2}}\left\|\nabla \omega_{x}\right\|^{\frac{1}{2}}\left\|j_{y}\right\|^{\frac{1}{2}}\left\|\nabla j_{y}\right\|^{\frac{1}{2}} \\
\leq & \frac{1}{62}\left\|\nabla \omega_{x}\right\|^{2}+\frac{1}{48}\left\|\nabla j_{y}\right\|^{2}+C\left(\|j\|^{2}+\left\|j_{y}\right\|^{2}\right)\left(\|\nabla \omega\|^{2}+\|\nabla j\|^{2}\right), \\
R_{13}^{3}= & \int \partial_{z} b_{3} \partial_{z} j_{j} \partial_{z} \omega_{j} \mathrm{~d} x \\
= & -\int\left(\partial_{x} b_{1}+\partial_{y} b_{2}\right) \partial_{z} j_{j} \partial_{z} \omega_{j} \mathrm{~d} x \\
\leq & C\left\|b_{x}\right\|^{\frac{1}{2}}\|\nabla j\|^{\frac{1}{2}}\|\nabla \omega\|^{\frac{1}{2}}\left\|\nabla \omega_{x}\right\|^{\frac{1}{2}}\left\|\nabla j_{y}\right\|^{\frac{1}{2}}\left\|j_{x}\right\|^{\frac{1}{2}} \\
& +C\left\|b_{y}\right\|^{\frac{1}{2}}\|\nabla j\|^{\frac{1}{2}}\|\nabla \omega\|^{\frac{1}{2}}\left\|\nabla \omega_{x}\right\|^{\frac{1}{2}}\left\|\nabla j_{y}\right\|^{\frac{1}{2}}\left\|j_{y}\right\|^{\frac{1}{2}} \\
\leq & \frac{1}{62}\left\|\nabla \omega_{x}\right\|^{2}+\frac{1}{48}\left\|\nabla j_{y}\right\|^{2}+C\left(\|j\|^{2}+\left\|j_{y}\right\|^{2}\right)\left(\|\nabla \omega\|^{2}+\|\nabla j\|^{2}\right) .
\end{aligned}
$$

Now, we turn to $R_{2}$,

$$
\begin{aligned}
R_{2} & =\int \partial_{k} b_{i} \partial_{i} \omega_{j} \partial_{k} j_{j} \mathrm{~d} x \\
& =\int \partial_{x} b_{i} \partial_{i} \omega_{j} \partial_{x} j_{j} \mathrm{~d} x+\int \partial_{y} b_{i} \partial_{i} \omega_{j} \partial_{y} j_{j} \mathrm{~d} x+\int \partial_{z} b_{i} \partial_{i} \omega_{j} \partial_{z} j_{j} \mathrm{~d} x=R_{21}+R_{22}+R_{23} .
\end{aligned}
$$


Thus, similarly, we deduce that

$$
\begin{aligned}
R_{21} & =\int \partial_{x} b_{i} \partial_{i} \omega_{j} \partial_{x} j_{j} \mathrm{~d} x \leq C\left\|b_{x}\right\|^{\frac{1}{2}}\|\nabla \omega\|^{\frac{1}{2}}\left\|j_{x}\right\|^{\frac{1}{2}}\left\|\nabla \omega_{x}\right\|^{\frac{1}{2}}\left\|\nabla j_{x}\right\|^{\frac{1}{2}}\left\|j_{x}\right\|^{\frac{1}{2}} \\
& \leq \frac{1}{62}\left\|\nabla \omega_{x}\right\|^{2}+\frac{1}{50}\left\|\nabla j_{x}\right\|^{2}+C\left(\left\|b_{x}\right\|^{2}+\left\|j_{x}\right\|^{2}\right)\left(\|\nabla \omega\|^{2}+\|\nabla j\|^{2}\right), \\
R_{22} & =\int \partial_{y} b_{i} \partial_{i} \omega_{j} \partial_{y} j_{j} \mathrm{~d} x \leq C\left\|b_{y}\right\|^{\frac{1}{2}}\|\nabla \omega\|^{\frac{1}{2}}\left\|j_{y}\right\|^{\frac{1}{2}}\left\|\nabla \omega_{x}\right\|^{\frac{1}{2}}\left\|\nabla j_{y}\right\|^{\frac{1}{2}}\left\|j_{y}\right\|^{\frac{1}{2}} \\
& \leq \frac{1}{62}\left\|\nabla \omega_{x}\right\|^{2}+\frac{1}{48}\left\|\nabla j_{y}\right\|^{2}+C\left(\left\|b_{y}\right\|^{2}+\left\|j_{y}\right\|^{2}\right)\left(\|\nabla \omega\|^{2}+\|\nabla j\|^{2}\right) .
\end{aligned}
$$

For $R_{23}$, we have

$$
\begin{aligned}
R_{23} & =\int \partial_{z} b_{i} \partial_{i} \omega_{j} \partial_{z} j_{j} \mathrm{~d} x \\
& =\int \partial_{z} b_{1} \partial_{x} \omega_{j} \partial_{z} j_{j} \mathrm{~d} x+\int \partial_{z} b_{2} \partial_{y} \omega_{j} \partial_{z} j_{j} \mathrm{~d} x+\int \partial_{z} b_{3} \partial_{z} \omega_{j} \partial_{z} j_{j} \mathrm{~d} x \\
& =R_{23}^{1}+R_{23}^{2}+R_{23}^{3} .
\end{aligned}
$$

Similarly,

$$
\begin{aligned}
R_{23}^{1}= & \int \partial_{z} b_{1} \partial_{x} \omega_{j} \partial_{z} j_{j} \mathrm{~d} x \leq C\|j\|^{\frac{1}{2}}\left\|\omega_{x}\right\|^{\frac{1}{2}}\|\nabla j\|^{\frac{1}{2}}\left\|\nabla \omega_{x}\right\|^{\frac{1}{2}}\left\|\nabla j_{x}\right\|^{\frac{1}{2}}\left\|j_{y}\right\|^{\frac{1}{2}} \\
\leq & \frac{1}{62}\left\|\nabla \omega_{x}\right\|^{2}+\frac{1}{50}\left\|\nabla j_{x}\right\|^{2}+C\left(\|j\|^{2}+\left\|\omega_{x}\right\|^{2}\right)\|\nabla j\|^{2}, \\
R_{23}^{2}= & \int \partial_{z} b_{2} \partial_{y} \omega_{j} \partial_{z} j_{j} \mathrm{~d} x \leq C\|j\|^{\frac{1}{2}}\left\|\omega_{x}\right\|^{\frac{1}{2}}\|\nabla j\|^{\frac{1}{2}}\left\|\nabla j_{x}\right\|^{\frac{1}{2}}\left\|j_{y}\right\|^{\frac{1}{2}}\left\|\nabla \omega_{y}\right\|^{\frac{1}{2}} \\
\leq & \frac{1}{50}\left\|\nabla j_{x}\right\|^{2}+\frac{1}{40}\left\|\nabla \omega_{y}\right\|^{2}+C\left(\|j\|^{2}+\left\|j_{y}\right\|^{2}\right)\left(\|\nabla \omega\|^{2}+\|\nabla j\|^{2}\right), \\
R_{23}^{3}= & \int \partial_{z} b_{3} \partial_{z} \omega_{j} \partial_{z} j_{j} \mathrm{~d} x \\
= & -\int\left(\partial_{x} b_{1}+\partial_{y} b_{2}\right) \partial_{z} \omega_{j} \partial_{z} j_{j} \mathrm{~d} x \\
\leq & C\left\|b_{x}\right\|^{\frac{1}{2}}\|\nabla \omega\|^{\frac{1}{2}}\|\nabla j\|^{\frac{1}{2}}\left\|\nabla \omega_{x}\right\|^{\frac{1}{2}}\left\|\nabla j_{y}\right\|^{\frac{1}{2}}\left\|j_{x}\right\|^{\frac{1}{2}} \\
& +C\left\|b_{y}\right\|^{\frac{1}{2}}\|\nabla \omega\|^{\frac{1}{2}}\|\nabla j\|^{\frac{1}{2}}\left\|\nabla \omega_{x}\right\|^{\frac{1}{2}}\left\|\nabla j_{y}\right\|^{\frac{1}{2}}\left\|j_{y}\right\|^{\frac{1}{2}} \\
\leq & \frac{1}{62}\left\|\nabla \omega_{x}\right\|^{2}+\frac{1}{48}\left\|\nabla j_{y}\right\|^{2}+C\left(\left\|b_{x}\right\|^{2}+\left\|b_{y}\right\|^{2}+\left\|j_{x}\right\|^{2}+\left\|j_{y}\right\|^{2}\right)\left(\|\nabla \omega\|^{2}+\|\nabla j\|^{2}\right) .
\end{aligned}
$$

Finally, for the last term $S$, we have

$$
\begin{aligned}
S & =-\int \varepsilon_{i j k}\left(\partial_{j} b_{l} \partial_{l} u_{k}-\partial_{j} u_{l} \partial_{l} b_{k}\right) \Delta j_{i} \mathrm{~d} x \\
& =\int \varepsilon_{i j k} \partial_{m}\left(\partial_{j} b_{l} \partial_{l} u_{k}-\partial_{j} u_{l} \partial_{l} b_{k}\right) \partial_{m} j_{i} \mathrm{~d} x \\
& =S_{1}+S_{2} .
\end{aligned}
$$


We first consider $S_{1}$,

$$
\begin{aligned}
S_{1} & =\int \varepsilon_{i j k} \partial_{m}\left(\partial_{j} b_{l} \partial_{l} u_{k}\right) \partial_{m} j_{i} \mathrm{~d} x \\
& =\int \varepsilon_{i j k} \partial_{x}\left(\partial_{j} b_{l} \partial_{l} u_{k}\right) \partial_{x} j_{i} \mathrm{~d} x+\int \varepsilon_{i j k} \partial_{y}\left(\partial_{j} b_{l} \partial_{l} u_{k}\right) \partial_{y} j_{i} \mathrm{~d} x+\int \varepsilon_{i j k} \partial_{z}\left(\partial_{j} b_{l} \partial_{l} u_{k}\right) \partial_{z} j_{i} \mathrm{~d} x \\
& =S_{11}+S_{12}+S_{13} .
\end{aligned}
$$

We deduce that

$$
\begin{aligned}
S_{11}= & \int \varepsilon_{i j k} \partial_{x}\left(\partial_{j} b_{l} \partial_{l} u_{k}\right) \partial_{x} j_{i} \mathrm{~d} x=\int \varepsilon_{i j k} \partial_{x} \partial_{j} b_{l} \partial_{l} u_{k} \partial_{x} j_{i} \mathrm{~d} x+\int \varepsilon_{i j k} \partial_{j} b_{l} \partial_{x} \partial_{l} u_{k} \partial_{x} j_{i} \mathrm{~d} x \\
\leq & C\left\|j_{x}\right\|^{\frac{1}{2}}\|\omega\|^{\frac{1}{2}}\left\|j_{x}\right\|^{\frac{1}{2}}\left\|\omega_{x}\right\|^{\frac{1}{2}}\left\|\nabla j_{x}\right\|^{\frac{1}{2}}\left\|\nabla j_{y}\right\|^{\frac{1}{2}} \\
& +C\|j\|^{\frac{1}{2}}\left\|\omega_{x}\right\|^{\frac{1}{2}}\left\|j_{x}\right\|^{\frac{1}{2}}\left\|j_{x}\right\|^{\frac{1}{2}}\left\|\nabla \omega_{x}\right\|^{\frac{1}{2}}\left\|\nabla j_{x}\right\|^{\frac{1}{2}} \\
\leq & \frac{1}{62}\left\|\nabla \omega_{x}\right\|^{2}+\frac{1}{50}\left\|\nabla j_{x}\right\|^{2}+\frac{1}{48}\left\|\nabla j_{y}\right\|^{2}+C\left(\left\|j_{x}\right\|^{2}+\|\omega\|^{2}+\|j\|^{2}\right)\left(\|\nabla \omega\|^{2}+\|\nabla j\|^{2}\right), \\
S_{12}= & \int \varepsilon_{i j k} \partial_{y}\left(\partial_{j} b_{l} \partial_{l} u_{k}\right) \partial_{y} j_{i} \mathrm{~d} x=\int \varepsilon_{i j k} \partial_{y} \partial_{j} b_{l} \partial_{l} u_{k} \partial_{y} j_{i} \mathrm{~d} x+\int \varepsilon_{i j k} \partial_{j} b_{l} \partial_{y} \partial_{l} u_{k} \partial_{y} j_{i} \mathrm{~d} x \\
\leq & C\left\|j_{y}\right\|^{\frac{1}{2}}\|\omega\|^{\frac{1}{2}}\left\|j_{y}\right\|^{\frac{1}{2}}\left\|\nabla j_{x}\right\|^{\frac{1}{2}}\left\|\nabla j_{y}\right\|^{\frac{1}{2}}\|\nabla \omega\|^{\frac{1}{2}} \\
& +C\|j\|^{\frac{1}{2}}\left\|\omega_{y}\right\|^{\frac{1}{2}}\left\|j_{y}\right\|^{\frac{1}{2}}\left\|j_{x}\right\|^{\frac{1}{2}}\left\|\nabla \omega_{y}\right\|^{\frac{1}{2}}\left\|\nabla j_{x}\right\|^{\frac{1}{2}} \\
\leq & \frac{1}{40}\left\|\nabla \omega_{y}\right\|^{2}+\frac{1}{50}\left\|\nabla j_{x}\right\|^{2}+\frac{1}{48}\left\|\nabla j_{y}\right\|^{2} \\
& +C\left(\|\omega\|^{2}+\left\|j_{y}\right\|^{2}+\|j\|^{2}+\left\|j_{x}\right\|^{2}\right)\left(\|\nabla \omega\|^{2}+\|\nabla j\|^{2}\right) .
\end{aligned}
$$

As for $S_{13}$, we see that

$$
\begin{aligned}
S_{13}= & \int \varepsilon_{i j k} \partial_{z}\left(\partial_{j} b_{l} \partial_{l} u_{k}\right) \partial_{z} j_{i} \mathrm{~d} x \\
= & \int \varepsilon_{i j k} \partial_{z} \partial_{j} b_{l} \partial_{l} u_{k} \partial_{z} j_{i} \mathrm{~d} x+\int \varepsilon_{i j k} \partial_{j} b_{l} \partial_{z} \partial_{l} u_{k} \partial_{z} j_{i} \mathrm{~d} x \\
= & \int \varepsilon_{i j k} \partial_{z} \partial_{x} b_{l} \partial_{l} u_{k} \partial_{z} j_{i} \mathrm{~d} x+\int \varepsilon_{i j k} \partial_{z} \partial_{y} b_{l} \partial_{l} u_{k} \partial_{z} j_{i} \mathrm{~d} x+\int \varepsilon_{i j k} \partial_{z} \partial_{z} b_{l} \partial_{l} u_{k} \partial_{z} j_{i} \mathrm{~d} x \\
& +\int \varepsilon_{i j k} \partial_{x} b_{l} \partial_{z} \partial_{l} u_{k} \partial_{z} j_{i} \mathrm{~d} x+\int \varepsilon_{i j k} \partial_{y} b_{l} \partial_{z} \partial_{l} u_{k} \partial_{z} j_{i} \mathrm{~d} x+\int \varepsilon_{i j k} \partial_{z} b_{l} \partial_{z} \partial_{l} u_{k} \partial_{z} j_{i} \mathrm{~d} x \\
= & S_{13}^{1}+S_{13}^{2}+S_{13}^{3}+S_{13}^{4}+S_{13}^{5}+S_{13}^{6} .
\end{aligned}
$$

We deduce each term step by step as follows:

$$
\begin{aligned}
S_{13}^{1} & =\int \varepsilon_{i j k} \partial_{z} \partial_{x} b_{l} \partial_{l} u_{k} \partial_{z} j_{i} \mathrm{~d} x \\
& \leq C\left\|j_{x}\right\|^{\frac{1}{2}}\|\omega\|^{\frac{1}{2}}\|\nabla j\|^{\frac{1}{2}}\left\|\nabla j_{y}\right\|^{\frac{1}{2}}\left\|\omega_{x}\right\|^{\frac{1}{2}}\left\|\nabla j_{x}\right\|^{\frac{1}{2}} \\
& \leq \frac{1}{50}\left\|\nabla j_{x}\right\|^{2}+\frac{1}{48}\left\|\nabla j_{y}\right\|^{2}+C\left(\|\omega\|^{2}+\left\|j_{x}\right\|^{2}\right)\left(\|\nabla \omega\|^{2}+\|\nabla j\|^{2}\right),
\end{aligned}
$$




$$
\begin{aligned}
S_{13}^{2} & =\int \varepsilon_{i j k} \partial_{z} \partial_{y} b_{l} \partial_{l} u_{k} \partial_{z} j_{i} \mathrm{~d} x \\
& \leq C\left\|j_{y}\right\|^{\frac{1}{2}}\|\omega\|^{\frac{1}{2}}\|\nabla j\|^{\frac{1}{2}}\left\|\nabla j_{x}\right\|^{\frac{1}{2}}\left\|\omega_{y}\right\|^{\frac{1}{2}}\left\|\nabla j_{y}\right\|^{\frac{1}{2}} \\
& \leq \frac{1}{50}\left\|\nabla j_{x}\right\|^{2}+\frac{1}{48}\left\|\nabla j_{y}\right\|^{2}+C\left(\|\omega\|^{2}+\left\|j_{y}\right\|^{2}\right)\left(\|\nabla \omega\|^{2}+\|\nabla j\|^{2}\right) .
\end{aligned}
$$

For $S_{13}^{3}$, we have

$$
\begin{aligned}
S_{13}^{3} & =\int \varepsilon_{i j k} \partial_{z} \partial_{z} b_{l} \partial_{l} u_{k} \partial_{z} j_{i} \mathrm{~d} x \\
& =\int \varepsilon_{i j k} \partial_{z} \partial_{z} b_{1} \partial_{x} u_{k} \partial_{z} j_{i} \mathrm{~d} x+\int \varepsilon_{i j k} \partial_{z} \partial_{z} b_{2} \partial_{y} u_{k} \partial_{z} j_{i} \mathrm{~d} x+\int \varepsilon_{i j k} \partial_{z} \partial_{z} b_{3} \partial_{z} u_{k} \partial_{z} j_{i} \mathrm{~d} x \\
& =S_{13}^{31}+S_{13}^{32}+S_{13}^{33} .
\end{aligned}
$$

Thus, we deduce that

$$
\begin{aligned}
& S_{13}^{31}= \int \varepsilon_{i j k} \partial_{z} \partial_{z} b_{1} \partial_{x} u_{k} \partial_{z} j_{i} \mathrm{~d} x \\
& \leq C\|\nabla j\|^{\frac{1}{2}}\left\|u_{x}\right\|^{\frac{1}{2}}\|\nabla j\|^{\frac{1}{2}}\left\|\nabla j_{x}\right\|^{\frac{1}{2}}\left\|\nabla j_{y}\right\|^{\frac{1}{2}}\left\|\omega_{x}\right\|^{\frac{1}{2}} \\
& \leq \frac{1}{50}\left\|\nabla j_{x}\right\|^{2}+\frac{1}{48}\left\|\nabla j_{y}\right\|^{2}+C\left(\left\|u_{x}\right\|^{2}+\left\|\omega_{x}\right\|^{2}\right)\|\nabla j\|^{2}, \\
& S_{13}^{32}= \int \varepsilon_{i j k} \partial_{z} \partial_{z} b_{2} \partial_{y} u_{k} \partial_{z} j_{i} \mathrm{~d} x \\
& \leq C\|\nabla j\|^{\frac{1}{2}}\left\|u_{y}\right\|^{\frac{1}{2}}\|\nabla j\|^{\frac{1}{2}}\left\|\nabla j_{x}\right\|^{\frac{1}{2}}\left\|\nabla j_{y}\right\|^{\frac{1}{2}}\left\|\omega_{x}\right\|^{\frac{1}{2}} \\
& \leq \frac{1}{50}\left\|\nabla j_{x}\right\|^{2}+\frac{1}{48}\left\|\nabla j_{y}\right\|^{2}+C\left(\left\|u_{y}\right\|^{2}+\left\|\omega_{y}\right\|^{2}\right)\|\nabla j\|^{2}, \\
& S_{13}^{33}= \int \varepsilon_{i j k} \partial_{z} \partial_{z} b_{3} \partial_{z} u_{k} \partial_{z} j_{i} \mathrm{~d} x \\
&=-\int \varepsilon_{i j k} \partial_{z}\left(\partial_{x} b_{1}+\partial_{y} b_{2}\right) \partial_{z} u_{k} \partial_{z} j_{i} \mathrm{~d} x \\
& \leq C\left\|j_{x}\right\|^{\frac{1}{2}}\|\omega\|^{\frac{1}{2}}\|\nabla j\|^{\frac{1}{2}}\left\|\nabla j_{x}\right\|^{\frac{1}{2}}\left\|\nabla j_{y}\right\|^{\frac{1}{2}}\|\nabla \omega\|^{\frac{1}{2}} \\
&+C\left\|j_{y}\right\|^{\frac{1}{2}}\|\omega\|^{\frac{1}{2}}\|\nabla j\|^{\frac{1}{2}}\left\|\nabla j_{x}\right\|^{\frac{1}{2}}\left\|\nabla j_{y}\right\|^{\frac{1}{2}}\|\nabla \omega\|^{\frac{1}{2}} \\
& \leq \frac{1}{50}\left\|\nabla j_{x}\right\|^{2}+\frac{1}{48}\left\|\nabla j_{y}\right\|^{2}+C\left(\|\omega\|^{2}+\left\|j_{x}\right\|^{2}+\left\|j_{y}\right\|^{2}\right)\left(\|\nabla \omega\|^{2}+\|\nabla j\|^{2}\right) \\
& \leq C\left\|b_{y}\right\|^{\frac{1}{2}}\|\nabla \omega\|^{\frac{1}{2}}\|\nabla j\|^{\frac{1}{2}}\left\|\nabla \omega_{x}\right\|^{\frac{1}{2}}\left\|\nabla j_{y}\right\|^{\frac{1}{2}}\left\|j_{y}\right\|^{\frac{1}{2}} \\
& \leq \frac{1}{62}\left\|\nabla \omega_{x}\right\|^{2}+\frac{1}{48}\left\|\nabla j_{y}\right\|^{2}+C\left(\left\|b_{y}\right\|^{2}+\left\|j_{y}\right\|^{2}\right)\left(\|\nabla \omega\|^{2}+\|\nabla j\|^{2}\right) \\
& S_{13}^{4}= \int \varepsilon_{i j k} \partial_{x} b_{l} \partial_{z} \partial_{l} u_{k} \partial_{z} j_{i} \mathrm{~d} x \\
& \leq C\left\|b_{x}\right\|^{\frac{1}{2}}\|\nabla \omega\|^{\frac{1}{2}}\|\nabla j\|^{\frac{1}{2}}\left\|\nabla \omega_{x}\right\|^{\frac{1}{2}}\left\|\nabla j_{y}\right\|^{\frac{1}{2}}\left\|j_{x}\right\|^{\frac{1}{2}} \\
& \leq \frac{1}{62}\left\|\nabla \omega_{x}\right\|^{2}+\frac{1}{48}\left\|\nabla j_{y}\right\|^{2}+C\left(\left\|b_{x}\right\|^{2}+\left\|j_{x}\right\|^{2}\right)\left(\|\nabla \omega\|^{2}+\|\nabla j\|^{2}\right), \\
& j_{j} \mathrm{~d} x
\end{aligned}
$$


For the last term $S_{13}^{6}$, we see that

$$
\begin{aligned}
S_{13}^{6} & =\int \varepsilon_{i j k} \partial_{z} b_{l} \partial_{z} \partial_{l} u_{k} \partial_{z} j_{i} \mathrm{~d} x \\
& =\int \varepsilon_{i j k} \partial_{z} b_{1} \partial_{z} \partial_{x} u_{k} \partial_{z} j_{i} \mathrm{~d} x+\int \varepsilon_{i j k} \partial_{z} b_{2} \partial_{z} \partial_{y} u_{k} \partial_{z} j_{i} \mathrm{~d} x+\int \varepsilon_{i j k} \partial_{z} b_{3} \partial_{z} \partial_{z} u_{k} \partial_{z} j_{i} \mathrm{~d} x \\
& =S_{13}^{61}+S_{13}^{62}+S_{13}^{63} .
\end{aligned}
$$

Then,

$$
\begin{aligned}
S_{13}^{61}= & \int \varepsilon_{i j k} \partial_{z} b_{1} \partial_{z} \partial_{x} u_{k} \partial_{z} j_{i} \mathrm{~d} x \leq C\|j\|^{\frac{1}{2}}\left\|\omega_{x}\right\|^{\frac{1}{2}}\|\nabla j\|^{\frac{1}{2}}\left\|\nabla j_{x}\right\|^{\frac{1}{2}}\left\|j_{y}\right\|^{\frac{1}{2}}\left\|\nabla \omega_{x}\right\|^{\frac{1}{2}} \\
\leq & \frac{1}{62}\left\|\nabla \omega_{x}\right\|^{2}+\frac{1}{50}\left\|\nabla j_{x}\right\|^{2}+C\left(\|j\|^{2}+\left\|\omega_{x}\right\|^{2}\right)\|\nabla j\|^{2}, \\
S_{13}^{62}= & \int \varepsilon_{i j k} \partial_{z} b_{2} \partial_{z} \partial_{y} u_{k} \partial_{z} j_{i} \mathrm{~d} x \leq C\|j\|\left\|^{\frac{1}{2}}\right\| \omega_{y}\left\|^{\frac{1}{2}}\right\| \nabla j\left\|^{\frac{1}{2}}\right\| \nabla j_{x}\left\|^{\frac{1}{2}}\right\| j_{y}\left\|^{\frac{1}{2}}\right\| \nabla \omega_{y} \|^{\frac{1}{2}} \\
\leq & \frac{1}{40}\left\|\nabla \omega_{y}\right\|^{2}+\frac{1}{50}\left\|\nabla j_{x}\right\|^{2}+C\left(\|j\|^{2}+\left\|\omega_{y}\right\|^{2}\right)\|\nabla j\|^{2}, \\
S_{13}^{63}= & \int \varepsilon_{i j k} \partial_{z} b_{3} \partial_{z} \partial_{z} u_{k} \partial_{z} j_{i} \mathrm{~d} x \\
= & -\int \varepsilon_{i j k}\left(\partial_{x} b_{1}+\partial_{y} b_{2}\right) \partial_{z} \partial_{z} u_{k} \partial_{z} j_{i} \mathrm{~d} x \\
\leq & C\left\|b_{x}\right\|^{\frac{1}{2}}\|\nabla \omega\|^{\frac{1}{2}}\|\nabla j\|^{\frac{1}{2}}\left\|\nabla \omega_{x}\right\|^{\frac{1}{2}}\left\|\nabla j_{y}\right\|^{\frac{1}{2}}\left\|j_{y}\right\|^{\frac{1}{2}} \\
& +C\left\|b_{y}\right\|^{\frac{1}{2}}\|\nabla \omega\|^{\frac{1}{2}}\|\nabla j\|^{\frac{1}{2}}\left\|\nabla \omega_{x}\right\|^{\frac{1}{2}}\left\|\nabla j_{y}\right\|^{\frac{1}{2}}\left\|j_{y}\right\|^{\frac{1}{2}} \\
\leq & \frac{1}{62}\left\|\nabla \omega_{x}\right\|^{2}+\frac{1}{48}\left\|\nabla j_{y}\right\|^{2}+C\left(\left\|b_{x}\right\|^{2}+\left\|b_{y}\right\|^{2}+\left\|j_{y}\right\|^{2}\right)\|\nabla j\|^{2} .
\end{aligned}
$$

As for $S_{2}$, deduced by similar methods, we can obtain the following inequality (for simplicity we omit the details here):

$$
\begin{aligned}
S_{2} \leq & \frac{1}{62}\left\|\nabla \omega_{x}\right\|^{2}+\frac{1}{40}\left\|\nabla \omega_{y}\right\|^{2}+\frac{1}{50}\left\|\nabla j_{x}\right\|^{2} \\
& +\frac{1}{48}\left\|\nabla j_{y}\right\|^{2}+\left(\left\|\omega_{x}\right\|^{2}+\|\omega\|^{2}+\|j\|^{2}+\left\|\omega_{y}\right\|^{2}\right. \\
& \left.+\left\|b_{x}\right\|^{2}+\left\|j_{x}\right\|^{2}+\left\|b_{y}\right\|^{2}+\left\|j_{y}\right\|^{2}+\left\|u_{x}\right\|^{2}+\left\|u_{y}\right\|^{2}+\left\|\omega_{y}\right\|^{2}\right)\left(\|\nabla \omega\|^{2}+\|\nabla j\|^{2}\right) .
\end{aligned}
$$

Then, substituting all the above estimates into (11), we finally deduce that

$$
\begin{aligned}
\frac{1}{2} \frac{\mathrm{d}}{\mathrm{d} t}\left(\|\nabla \omega\|^{2}+\|\nabla j\|^{2}\right)+\frac{1}{2}\left(\left\|\nabla \omega_{x}\right\|^{2}+\left\|\nabla \omega_{y}\right\|^{2}+\left\|\nabla j_{x}\right\|^{2}+\left\|\nabla j_{y}\right\|^{2}\right) \\
\leq C\left(\left\|\omega_{x}\right\|^{2}+\|\omega\|^{2}+\|j\|^{2}+\left\|\omega_{y}\right\|^{2}+\left\|b_{x}\right\|^{2}+\left\|j_{x}\right\|^{2}+\left\|b_{y}\right\|^{2}+\left\|j_{y}\right\|^{2}\right. \\
\left.\quad+\left\|u_{x}\right\|^{2}+\left\|u_{y}\right\|^{2}+\left\|\omega_{y}\right\|^{2}\right)\left(\|\nabla \omega\|^{2}+\|\nabla j\|^{2}\right) .
\end{aligned}
$$

Then we complete the proof of Lemma 2.3 by Gronwall's lemma. 


\section{Proof of Theorem 1.1}

In this section, we prove Theorem 1.1 by using the method of vanishing viscosity. To this end, we consider the following regularized problem:

$$
\begin{aligned}
& u_{t}^{\varepsilon}+u^{\varepsilon} \cdot \nabla u^{\varepsilon}=-\nabla p^{\varepsilon}+u_{x x}^{\varepsilon}+u_{y y}^{\varepsilon}+\varepsilon u_{z z}^{\varepsilon}+b^{\varepsilon} \cdot \nabla b^{\varepsilon}, \\
& b_{t}^{\varepsilon}+u^{\varepsilon} \cdot \nabla b^{\varepsilon}=b_{x x}^{\varepsilon}+b_{y y}^{\varepsilon}+\varepsilon b_{z z}^{\varepsilon}+b^{\varepsilon} \cdot \nabla u^{\varepsilon}, \\
& \operatorname{div} u^{\varepsilon}=0, \quad \operatorname{div} b^{\varepsilon}=0,
\end{aligned}
$$

with smooth initial data

$$
u^{\varepsilon}(0, x)=\psi_{\varepsilon} * u_{0}, \quad b^{\varepsilon}(0, x)=\psi_{\varepsilon} * b_{0},
$$

where $\psi_{\varepsilon}(x, y)=\varepsilon^{-2} \psi(x / \varepsilon, y / \varepsilon)$ is the standard mollifier satisfying

$$
\psi \geq 0, \quad \psi \in C_{0}^{\infty}\left(\mathbb{R}^{3}\right) \text { and } \int \psi \mathrm{d} x=1
$$

Now, an application of the classical result shows that for any $T>0$, there exists a unique global smooth solution $\left(u^{\varepsilon}, b^{\varepsilon}\right)$ of (12)-(15) on $\mathbb{R}^{3} \times(0, T)$ satisfying the global bounds stated in Lemma 2.2 and 2.3, which are uniform in $\varepsilon$. So, by standard compactness arguments, we can extract a subsequence $\left(u^{\varepsilon_{j}}, b^{\varepsilon_{j}}\right)$ and pass to the limit as $j \rightarrow \infty$ to get that the limit function $(u, b)$ is indeed a global smooth solution of the problem (1)-(4). The proof of Theorem 1.1 is therefore completed.

\section{Competing interests}

The authors declare that they have no competing interests.

Authors' contributions

MS carried out the main work and drafted the manuscript. JW participated in completing the proof of Lemma 2.2. All authors read and approved the final manuscript.

\section{Author details}

${ }^{1}$ School of Mathematical Sciences, Luoyang Normal University, Luoyang, 471022, P.R. China. ${ }^{2}$ Key Laboratory of Symbolic Computation and Knowledge Engineering of Ministry of Education, Jilin University, Changchun, 130012, P.R. China.

${ }^{3}$ School of Mathematical Sciences, Ocean University of China, Qingdao, 266000, P.R. China.

\section{Acknowledgements}

This work was supported by the National Nature Science Foundation of China (No.11026079), the Youth Backbone Teacher Foundation of Henan Province (No. 2010GGJS-167), the Natural Science Foundation of Henan Province (No.122300410261), and the Foundation of Laboratory of Symbolic Computation and Knowledge Engineering of Ministry of Education (No. 93K172012K07)

Received: 7 January 2013 Accepted: 10 July 2013 Published: 26 July 2013

\section{References}

1. Wang, F, Wang, K: Global existence of 3D MHD equations with mixed partial dissipation and magnetic diffusion. Nonlinear Anal., Real World Appl. 14, 525-536 (2013)

2. Cabannes, H, Theoretical Magnetofluiddynamics. Academic Press, New York (1970)

3. Cao, C, Wu, J: Global regularity for the 2D MHD equations with mixed partial dissipation and magnetic diffusion. Adv. Math. 226, 1803-1822 (2011)

doi:10.1186/1029-242X-2013-345

Cite this article as: Su and Wang: Global smooth solutions to 3D MHD with mixed partial dissipation and magnetic diffusion. Journal of Inequalities and Applications 2013 2013:345. 